\title{
ON CHARACTERS IN THE PRINCIPAL 2-BLOCK
}

\author{
THOMAS R. BERGER AND MARCEL HERZOG
}

(Received 5 August 1976)

\begin{abstract}
Let $k$ be a complex number and let $u$ be an element of a finite group $G$. Suppose that $u$ does not belong to $O(G)$, the maximal normal subgroup of $G$ of odd order. It is shown that $G$ satisfies $X(1)-X(u)=k$ for every complex nonprincipal irreducible character $X$ in the principal 2-block of $G$ if and only if $G / O(G)$ is isomorphic either to $C_{2}$, a cyclic group of order 2, or to $\operatorname{PSL}\left(2,2^{n}\right)$, $n \geqq 2$.
\end{abstract}

Let $G$ be a finite group. It was shown by Kwok (1975) that if $u \in G^{*}$ satisfies

$$
X(1)-X(u)=\dot{k}
$$

for every complex nonprincipal irreducible character $X$ of $G$, then a Sylow 2-subgroup of $G$ is elementary abelian. Moreover, if $G$ is simple, then $G \cong P S L\left(2,2^{n}\right)$. A complex characterization of such groups is given in Herzog (1976). A more general equality is analyzed in Herzog (to appear).

The aim of this paper is to classify groups satisfying (1) for every complex nonprincipal irreducible character in the principal 2-block of $G$. We prove:

THEOREM. Let $G$ be a finite group, $u$ an element of $G$ and $k$ a complex number. Suppose that (1) is satisfied by every complex nonprincipal irreducible character of $G$ belonging to $B$, the principal 2-block of $G$.

Then one of the following statements holds:

(a) $u \in O(G)$,

(b) $|G / O(G)|=2, u \notin O(G)$, or

(c) $G / O(G) \cong P S L\left(2,2^{n}\right), n \geqq 2, u \notin O(G)$ but $u^{2} \in O(G)$.

Conversely, if $G$ and $u$ satisfy $(a),(b)$ or $(c)$, then (1) holds.

PROOF. It is well known for the principal block that

$$
O(G)=\cap\left\{\operatorname{ker} X \mid X \in B^{*}\right\}
$$

where $B^{*}$ denotes $B \backslash 1_{G}$. Thus (1) holds with $k=0$ if and only if $u \in O(G)$. 
It is easy to check that if $G$ and $u$ satisfy (b) or (c), then (1) holds. Consequently, it suffices to show that if

(i) $O(G)=1, u \neq 1$ (hence $k \neq 0$ ) and (1) holds, then either (b) or (c) is satisfied.

From now on we denote by $\Sigma$ or $\Sigma^{*}$ the summation over all $X \in B$ or $X \in B^{*}$, respectively.

By (i) $g=|G|$ is even. If $z$ is an involution in $G$, then, by the orthogonality relations in blocks (O.R.B.), $\Sigma X(1) X(z)=0$. Since $X(z) \equiv$ $X(1)(\bmod 2), 1_{G}(1) 1_{G}(z)=1$, and $X(1)^{2} \equiv X(1)(\bmod 2)$, it follows that

(ii) $\Sigma^{*} X(1)$ is odd.

As $\Sigma^{*} X(u)$ is a rational integer, (1) implies that $k$ is both rational and an algebraic integer, therefore,

(iii) $k$ is a positive integer.

Suppose that $y \in G$ lies outside the 2-sections of 1 and $u$. Then by the O.R.B. we get, in view of (1);

$$
0=\Sigma X(y)(X(1)-X(u))=k \Sigma^{*} X(y),
$$

hence

$$
\Sigma^{*} X(y)=0 .
$$

Let $w$ be a 2-element of $G$ of maximal order and let $z$ be the involution in $\langle w\rangle$. Let $\mathscr{P}$ be a prime ideal lying over 2 in $O$, the integers in $Q(\sqrt[\&]{1})$. Since each of $X(w), X(z)$, and $X(1)$ is a sum of $X(1)$ 2-power roots of unity, we have

$$
X(w) \equiv X(z) \equiv X(1)(\bmod \mathscr{P}),
$$

hence by (ii)

$$
\Sigma^{*} X(w) \equiv \Sigma^{*} X(z) \equiv \Sigma^{*} X(1) \equiv 1(\bmod \mathscr{P}) .
$$

Thus, by (2) $w$ and $z$ belong to the 2-section of $u$. Consequently, if $S$ denotes a Sylow 2-subgroup of $G$, then:

(iv) $S$ is elementary abelian,

(v) $G$ has one class of involutions, and

(vi) $|u|=2 d, d$ odd.

Choose $H$, a minimal normal subgroup in $G$. Then, by (iv) and (v), $|G: H|$ is odd and $H$ is characteristically simple. Suppose that $H=$ $H_{1} \times \cdots \times H_{i}$, where $H_{i}$ is nonabelian simple and $t>1$. Let $x \in H_{1}, y \in H_{2}$ be involutions. By the Krull-Schmidt Theorem the components of $H$ are unique, so that, by conjugation, $G$ acts to permute the components $H_{i}$. Hence $x$ and $x y$ are nonconjugate involutions in $G$, contradicting (v). Thus we have proven 
that $H$ is either an elementary abelian 2-group or a nonabelian simple group with an elementary abelian Sylow 2-subgroup $S$. By Walter (1969), in the latter case $H$ is isomorphic to one of the following groups: $\operatorname{PSL}(2, q), q>3$, $q \equiv 0,3$ or $5(\bmod 8), J$ (Janko's smallest group) or $\operatorname{Re}(q)$ ( group of Ree type).

If $G=H$, then it is easy to check that either (b) or (c) holds. Thus assume, from now on, that

(vii) $G / H$ is a nontrivial solvable group of odd order.

Let $Y$ be a nonprincipal linear character of $G / H$ and suppose that $Y \in B$. Then as $Y(1)=1$, by (1) and (iii) $Y(u)=-1$, in contradiction to (vii). Thus:

(viii) no nonprincipal linear character of $G / H$ belongs to $B$.

By the Frattini argument $G=N(S) H$, hence $C_{G}(S) H \triangleleft G$. Suppose that $G \supset C_{G}(S) H$; then by the solvability of $G / C_{G}(S) H, G^{\prime} C_{G}(S) H \subset G$. Let $M$ be a maximal (hence normal of prime index) subgroup of $G$ containing $G^{\prime} C_{G}(S) H$ and let $Y$ be a nonprincipal linear character of $G / M$. By (viii) $Y \notin B$, hence by Brauer's criterion for block membership for some $x \in G$

$$
c Y(x) \not \equiv c 1_{G}(x)(\bmod \mathscr{P})
$$

where $c=\left|G: C_{G}(x)\right|$ and $\mathscr{P}$ is a prime ideal over 2 in $\mathcal{O}$, the integers of $Q(\xi / \overline{1})$. We conclude that $c$ is odd and $x \notin$ ker $Y=M$. Thus $G=\langle x\rangle M$ and $x \in C_{G}\left(S_{1}\right)$ for some Sylow 2-subgroup $S_{1}$ of $G$. As $M \supseteq C_{G}\left(S_{1}\right), G=M$, a contradiction. We have shown that

(ix) $G=C_{G}(S) H$.

If $H$ is a 2-group, then by Lemma 1.2.3 of Hall and Higman

$$
C_{G}(S) \subseteq S=H=G
$$

contradicting (vii). So assume, from now on, that

(x) $H$ is a nonabelian simple group.

Suppose that $x \in C_{G}(H)$; then $x$ is of odd order, hence $C_{G}(H) \subseteq$ $O(G)=1$. Thus

(xi) $G / H \tilde{C}$ Out $(H)$.

As Out $(J)=1$ (Janko, 1966), by (vii) $H \not J J$. If $H \cong P S L\left(2,2^{n}\right), n \geqq 2$, then $G$ is generated by $H$ and odd order field automorphisms of $H$ (Carter (1972), p. 211). Therefore these field automorphisms may be chosen to normalize and act faithfully on $S$, in contradiction to (ix). Since $\operatorname{PSL}(2,4) \cong$ PSL $(2,5)$, it remains to deal with the cases: $H \cong P S L(2, q), q>5, q \equiv 3$ or $5(\bmod 8)$ and $H \cong \operatorname{Re}(q)$.

First we prove, denoting by $\operatorname{Ir}(G / H)$ the set of the irreducible characters of $G / H$, that 
(xii) If $Y \in \operatorname{Irr}(G / H) \cap B$, then $Y=1_{G}$.

Suppose that $Y \neq 1_{G}$. Since $\bar{G}=G / H$ is of odd order $\bar{g}, Y$ does not belong to the principal 2-block of $\bar{G}$ and there exists $x \in C_{G}(S)$ such that, denoting $x H$ by $\bar{x}$, and using Brauer's criterion for block membership,

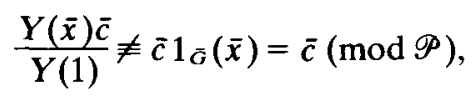

where $\bar{c}=\left|\bar{G}: C_{\bar{G}}(\bar{x})\right|$ and $\mathscr{P}$ is a prime ideal over 2 in $\mathcal{O}$, the integers of $Q(\sqrt[\&]{1})$. As $Y(1)$ and $\bar{c}$ are odd integers and $Y(\bar{x})=Y(x)$, it follows that

$$
Y(x) \not \equiv Y(1) \quad \text { hence } \frac{Y(x) c}{Y(1)} \not \equiv c(\bmod \mathscr{P}),
$$

where $c$ is the odd integer $\left|G: C_{G}(x)\right|$. Thus $Y \notin B$, a contradiction.

Suppose that $H \cong \operatorname{Re}(q)$. Simple groups of Ree type were described in Ward (1966), where their character table is given on pp. 87-88. We shall use his notation for $H$. Since $|G: H|$ is odd, it is easily seen from the character table of $\operatorname{Re}(q)$ that each of the 8 irreducible characters $\xi_{i}, i=1, \cdots, 8$ belonging to $B_{0}(H)$ (the principal 2-block of $H$ ), is stable in $G$. Thus if $X \in B$, then $\left.X\right|_{H}=e_{x} Y_{x}$, where $Y_{x} \in B_{0}(H)$ and $e_{x}$ is a positive integer. Consequently, if $h$ and $f$ denote elements of $H$ of even and odd order, respectively, we get by the O.R.B.:

$$
0=\Sigma X(h) X(f)=\Sigma e_{x}^{2} Y_{x}(h) Y_{x}(f)=\sum_{i=1}^{8} n_{i} \xi_{i}(h) \xi_{i}(f) .
$$

Clearly $n_{i} \geqq 1$ for $i=1, \cdots, 8$ and since by (xii) $1_{G}$ is the only element of $B$ with $H$ in its kernel, $n_{1}=1$. Again in the notation of Ward, choose $h=J R^{a} \neq J$ and $f=V$, so that by (3) $0=n_{1}-n_{3}$, hence $n_{3}=n_{1}=1$. Subsequent choices of $h=J R^{a} \neq J, J R^{a} \neq J, J T^{-1}, J T^{-1}$ and $f=Y, X, V, W$, respectively, yield $n_{2}=1, n_{4}=1, n_{5}=n_{7}=1$ and $n_{6}=n_{8}=1$, respectively, since 1, im $\sqrt{3 / 2}$ are rationally independent. It follows that $B$ consists of 8 characters $X_{i}, i=1, \cdots, 8$ such that $\left.X_{i}\right|_{H}=\xi_{i}, i=1, \cdots, 8$. Thus, by the O.R.B. and (1)

$$
\begin{aligned}
0 & =\Sigma X(u) X(W)=1-X_{3}(u)+X_{6}(u)+X_{8}(u) \\
& =1-X_{3}(1)+k+X_{6}(1)+X_{8}(1)-2 k,
\end{aligned}
$$

hence

$$
k=1-q^{3}+(q-1) m(q+1-3 m)<0
$$

a contradiction.

Finally, suppose that $H \cong P S L(2, q), q>5, q \equiv 3$ or $5(\bmod 8)$. The character table of $H$ is given in Ward (1966), p. 65. Since $|G: H|$ is odd, it is 
easy to see from his character table that each of the 4 irreducible characters $\theta_{i}$, $i=1, \cdots, 4$ belonging to $B_{0}(H)$ is stable in $G$. Thus we get a formula similar to (3) and by choosing $h=S_{0}^{(q-e) / 4}, S_{0}^{(q-e) / 4}$ and $f=R, T$, respectively, we get $1=n_{1}=n_{4}$ and $n_{2}=n_{3}=1$ since $(q-e) / 4$ is odd and $1, \sqrt{e q}$ are rationally independent. Hence $B$ consists of 4 characters $X_{i}, i=1, \cdots, 4$, such that $\left.X_{i}\right|_{H}=\theta_{i}, i=1, \cdots, 4$. Thus, by the O.R.B.,

$$
0=\Sigma X\left(R^{a}\right) X(u)=1-e X_{4}(u)
$$

hence $X_{4}(u)=e$ and by (1) $k=q-e$. As $q>5$, a contradiction is then reached by considering the equality $\Sigma X(1) X(u)=0$, completing the proof of the theorem.

\section{REFERENCES}

Roger W. Carter (1972), Simple Groups of Lie Type (John Wiley, New York).

Marcel Herzog (1976), 'On groups with extremal blocks', Bull. Austral. Math. Soc. 14, 325-330.

Marcel Herzog (to appear), 'On linear relations between character values', J. Algebra.

Zvonimir Janko (1966), 'A new finite simple group with abelian Sylow 2-subgroups and its characterization', J. Algebra 3, 147-186.

Chung-Mo Kwok (1975), 'A characterization of PSL (2,2m ), J. Algebra 34, 288-291.

John H. Walter (1969), 'The characterization of finite groups with abelian Sylow 2-subgroups', Ann. of Math. (2) 89, 405-514.

Harold N. Ward (1966), 'On Ree's series of simple groups', Trans. Amer. Math. Soc. 121, 62-89.

Department of Mathematics,

Institute of Advanced Studies,

The Australian National University,

Canberra, ACT 2600. 